1

\title{
Wideband Cavity-Backed CubeSat Antenna in S band
}

\author{
Miroslav J. Veljovic, Anja K. Skrivervik \\ Microwaves and Antennas Group (MAG), EPFL, Lausanne, Switzerland, miroslav.veljovic@epfl.ch
}

\begin{abstract}
The Telemetry, Tracking and Command (TT\&C) antennas are a crucial component of small satellites, as their in-orbit attitude is not always well-defined. The TT\&C antenna design for a CubeSat is an even more challenging task, considering the volume restrictions imposed by the standard, and the bandwidth requirements for a duplex communication. A detailed design process of a low profile S-band antenna, suitable for CubeSat applications, is presented in this paper. The fabricated prototype exhibits a 10-dB impedance bandwidth of $40 \%$, and a 3-dB axial-ratio bandwidth of $32 \%$.
\end{abstract}

Index Terms-Cavity-backed, wideband, stacked patch antenna, CubeSat, TT\&C.

\section{INTRODUCTION}

CubeSat is the most popular standard for small modular satellites. Each CubeSat is comprised of Units (U) - cubes having a size of $100 \times 100 \times 100 \mathrm{~mm}^{3}$ and a mass of up to $1.33 \mathrm{~kg}$ [1], with the most popular being $1 \mathrm{U}, 3 \mathrm{U}$ and $6 \mathrm{U}$ satellites [2]. The standard attracts many universities and industries with its low cost of building and orbital deployment. The small available volume and weight pose strict constraints on the system design, including the onboard antennas. During the launch, no feature on the satellite surface should protrude the volume designated by the standard, and either low-profile or deployable antenna structures are used [3].

Several antennas on board CubeSats realize various functions and their frequencies vary from VHF to $\mathrm{Ka}$ bands [3]. Among others, Telemetry, Tracking and Command (TT\&C) are vital for each satellite mission. Right after a satellite is detached from the launch vehicle, the attitude is not defined and wide-coverage antennas are required to establish a link with the ground stations and allow for subsequent satellite operations [4]. Therefore, the $\mathrm{TT} \& \mathrm{C}$ antenna reliability is crucial for a satellite mission success. In this sense, low-profile antennas are a favourable candidate over fail-prone deployable structures. On the other hand, size restrictions, primarily on the antenna thickness, result in limitations on the achievable impedance and axial ratio (AR) bandwidth with this kind of antennas, especially in the lower frequency bands. This is a known issue with planar antennas, and so far, only a few CubeSat antenna designs that yield narrowband AR performance are proposed for S-band frequencies [5], [6].

In this paper, we present a design of a wideband lowprofile circularly-polarized $(\mathrm{CP})$ cavity-backed patch antenna that fits on a single side of a $1 \mathrm{U}$ CubeSat and covers the Industrial, Scientific and Medical (ISM) $2.4 \mathrm{GHz}$ band and frequencies for Space Operation (SO), Space Research (SR) and Earth Exploration-Satellite services (EES) in the S band, as allocated by the European Cooperation for Space Standardization (ECSS). The initial aperture-coupled patch design, selected to acquire a better understanding of the antenna operation, is presented in Section II, including the measurement results of the first prototype. Section III describes a detailed set of modifications performed on the initial model, making it suitable for CubeSat applications. A prototype of the new cavity-backed stacked-patch antenna was built and validated. The measurement results are also presented in Section III. Potential limitations of this geometry and instructions for handling such limitations are described in Section IV.

\section{APERTURE-COUPLED PATCH ANTENNA}

The initial antenna requirements are summarized in Table 1.

An aperture-coupled patch antenna [7] was selected for a preliminary model, since this geometry provides the most bandwidth, compared with other methods of exciting patch antennas (coaxial pin, microstrip line). To better understand the operation of this antenna, a linearly-polarized (LP) microstrip-fed element was first tuned for the desired frequency band, having a minimal antenna thickness for the specified bandwidth. The circular polarization is then achieved using a crossed slot and a feeding network based on $\mathrm{T}$-junction power dividers, quarter-wavelength transformers and phase-delay lines. The design was carefully analysed in several steps, gradually increasing the feeding network complexity. Antenna geometry with intermediate design steps is shown in Fig 1.

The design was verified through a prototype shown in Fig. 2(a). RT Duroid 5870 substrate $(\varepsilon r=2.33, \tan \delta=0.0012$, $\mathrm{h}=0.508 \mathrm{~mm}$ ) is used for the feeding network and the coupling slot, the patch is etched on a thin FR4 sheet $(\varepsilon \mathrm{r}=4.55, \tan \delta=0.02, \mathrm{~h}=0.2 \mathrm{~mm})$ and separated from the slot using a space-eligible hard foam material $(\varepsilon \mathrm{r}=1.08$, $\tan \delta=0.0002, \mathrm{~h}=10 \mathrm{~mm})$. Individual layers are attached together using nylon screws.

TABLE I. INITIAL ANTENNA REQUIREMENTS

\begin{tabular}{|l|l|}
\hline Frequency band $[\mathrm{GHz}]$ & $2-2.45(\sim 20 \%)$ \\
\hline Antenna gain $[\mathrm{dBi}]$ & $>5$ \\
\hline Beamwidth $\left[^{\circ}\right]$ & $>50$ \\
\hline S11 $[\mathrm{dB}]$ & $<-10$ \\
\hline Axial Ratio $[\mathrm{dB}]$ & $<3$ \\
\hline Dimensions $\left[\mathrm{mm}^{3}\right]$ & $100 \times 100 \times 12$ \\
\hline
\end{tabular}




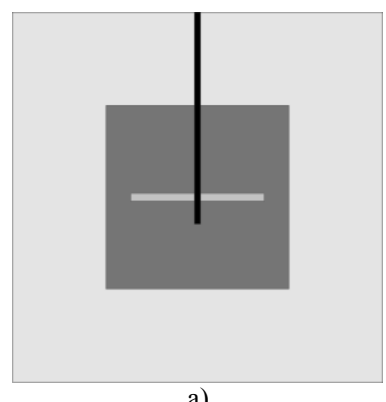

a)
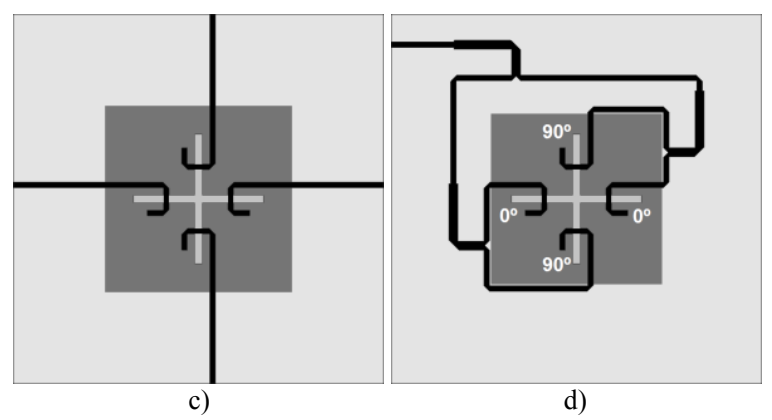

d)

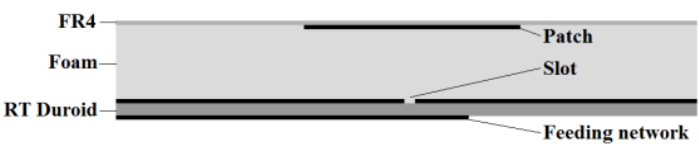

e)

Fig. 1. Aperture-coupled patch antenna model evolution. a) LP model 1. b) LP model 2. c) CP 4-port model. d) CP full model. e) Crosssection view. The figures show the feeding lines (black), the patch (dark grey) and the slots (light grey).

The prototype is characterized in an anechoic chamber, and the measured results are compared with the simulations in Fig. 2(b).

An intermediate 4-port model, that excludes the feeding network, is used to correctly evaluate the coupling to the radiating patch. The response of the 4-port model, shown in Fig. 2(b), is a good indicator of the power accepted by the patch in the desired polarization. The port excitation mimics the feeding network performance, and the active Sparameters ${ }^{(1)}$ are calculated in order to evaluate the reflection coefficient of the antenna in this configuration.

T-junctions are used in the full model as power dividers, thus multiple reflections exist inside the feeding network. The reflected signals ultimately arrive at the coupling slots with an unwanted phase shift and effectively contribute to the cross-polarization. This explains the low values of $S_{11}$ for the full model, shown in Fig. 2(b). Moreover, the similarity between the active- $\mathrm{S}_{1}$ of the 4-port model, and the AR of the full model seen in Fig. 2(c), confirms the previous analysis.

1 The active S-parameters of an N-port network are obtained using the following expression:

active $-\mathrm{S}_{\mathrm{m}}=\sum_{n=1}^{N} S_{m n} \frac{a_{n}}{a_{m}}, m=1, \ldots, N$,

where $S_{m n}$ are the conventional S-parameters. Parameter active- $\mathrm{S}_{\mathrm{m}}$ gives the reflected power in port $\mathrm{m}$ when all the ports are excited in a specified way (compared to the conventional S-parameters, where ports are excited one at a time).
The issue could be overcome if a better matching is achieved for a single element, which would reduce the reflections inside the network, or if Wilkinson dividers were used instead of T-junctions, in which case the reflections would be dissipated in the dividers' resistors. However, in this case, the active reflection coefficient of the antenna is limited by the available thickness, and T-junctions were selected as they allow a simpler manufacturing process.

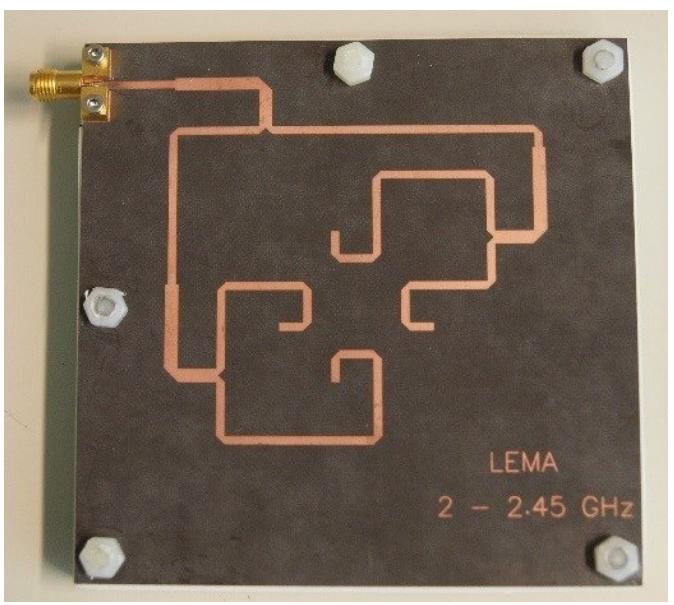

a)

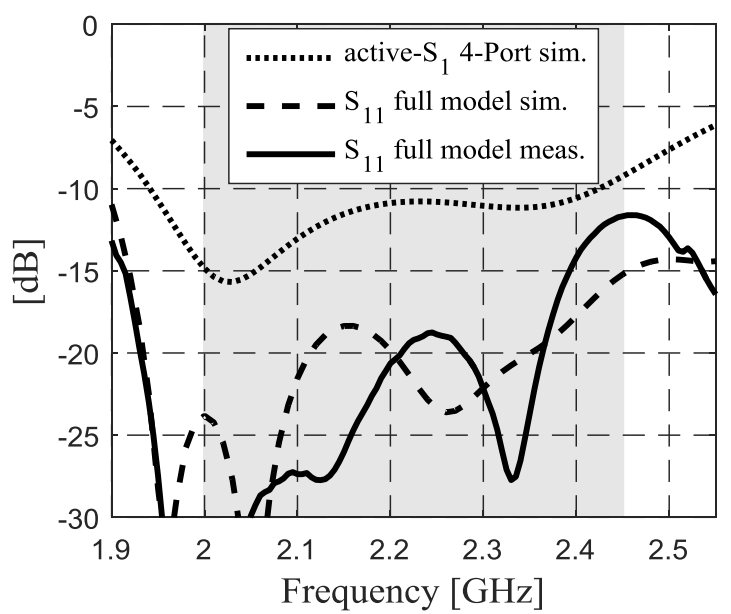

b)

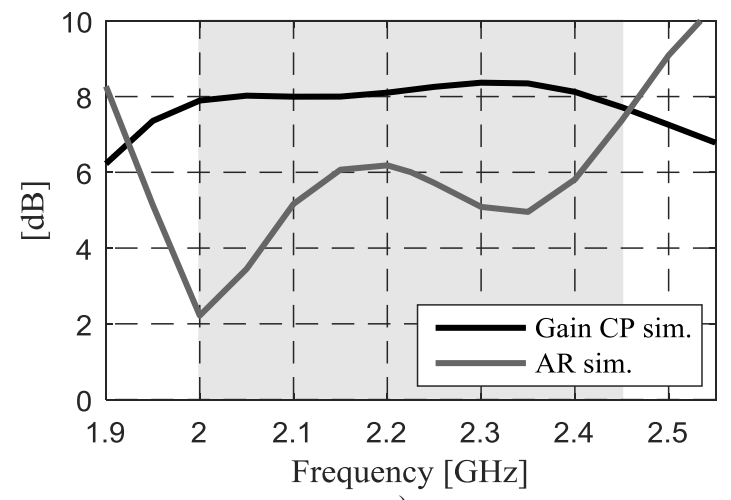

c)

Fig. 2. Aperture-coupled patch antenna. a) A realized prototype. b) The reflection coefficient. c) Simulated gain and axial ratio. 


\section{CAVITY-BACKED PATCH ANTENNA}

Once the antenna is mounted on the CubeSat, it is necessary to isolate it from the interior satellite components, in order to ensure a proper operation of both the antenna and on-board electronics. This can be accomplished by using a stripline-based feeding network. In this case, the electric field strength is equally distributed between the two ground planes in the substrate, which results in a poor coupling to the radiating patch. It is shown that the coupling to the patch is the critical point of this design, considering the bandwidth requirements and the thickness limitation. Several modifications were performed to improve it:

- A second, smaller patch was added below the original one, to improve the coupling at the higher frequencies. By adding the second patch, the total antenna thickness remains unchanged.

- Instead of a stripline, a layer of low-permittivity foam and a second ground plane were added above the microstrip lines. The resulting structure can be considered as an asymmetric stripline [8] and it focuses the electric fields in the dielectric layer close to the slot, ensuring a stronger coupling. This approach alone is, however, ineffective for a small ground plane separation that is required here, as the proximity of the second ground plane deteriorates the performance. This effect is demonstrated in Fig. 3.

- Mode-suppression pins were placed around the slot to reduce the power transferred to the parallel-plate mode in the stripline. The introduction of pins had an overall negative effect on the coupling since it perturbed the electric field distribution inside the stripline, in the vicinity of the slot. This effect was observed regardless of the number of pins, even if the density as high as $\lambda_{\mathrm{g}} / 10$ is used [9].

- Following the conclusions drawn from the previous point, the key modification was to enclose the whole feeding structure from all sides with a conductive layer, effectively creating a rectangular cavity in the asymmetric stripline. The electric field distribution inside the cavity has a maximum at the position of the slot, which drastically increases the power delivered to the radiating patch, and improves the overall antenna performance.

Other modifications are also possible, such as changing the shape of the slots, but they were not considered in this work.

A modified feeding network, based on Wilkinson power dividers and phase-delay lines, is used for the full antenna model, shown in Fig. 4. The result is a substantial improvement of the AR values, in comparison with the Tjunction-based feeding network, as the reflected signals are now absorbed by the dividers' resistors.

A prototype of the cavity-backed antenna was built to verify the design. The feeding asymmetric stripline consists of an RT Duroid 5870 substrate $(\varepsilon \mathrm{r}=2.33$, $\tan \delta=0.0012$, $\mathrm{h}=0.508 \mathrm{~mm}$ ) and a low-permittivity hard foam material $(\varepsilon \mathrm{r}=1.08, \tan \delta=0.0002, \mathrm{~h}=2 \mathrm{~mm})$.
A metallized FR4 is used as the second ground plane. The patches are etched on two other FR4 sheets $(\mathrm{h}=0.1 \mathrm{~mm})$ and separated using two hard foam layers $(\mathrm{h}=4 \mathrm{~mm}, \mathrm{~h}=5 \mathrm{~mm})$. All the layers are attached with nylon screws. The conductive shield around the stripline was first approximated with a pin wall, having the pin separation of $15.6 \mathrm{~mm}$ $(\sim 0.1 \lambda \mathrm{g}$, where $\lambda \mathrm{g}$ is the wavelength inside the stripline at 2 $\mathrm{GHz}$ ). The pins were manually soldered to the two ground planes, since metallized vias, or any kind of metallization at all, is not feasible on the foam material. The first measurements were performed in this configuration. Afterwards, the stripline was enclosed with a conductive tape, and the measurements were repeated.

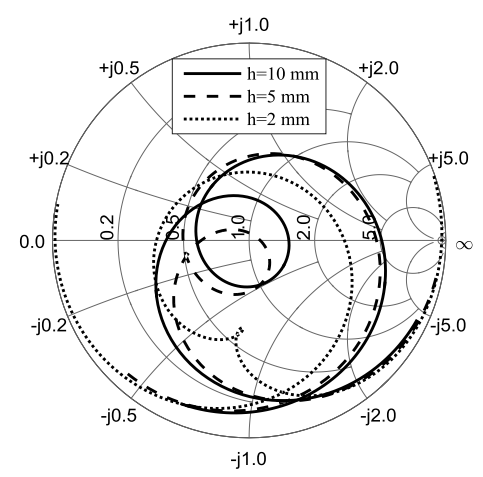

Fig. 3. Active S-parameter of the 4-port asymmetric-stripline model, for different separations of the second ground plane $(\mathrm{h})$.

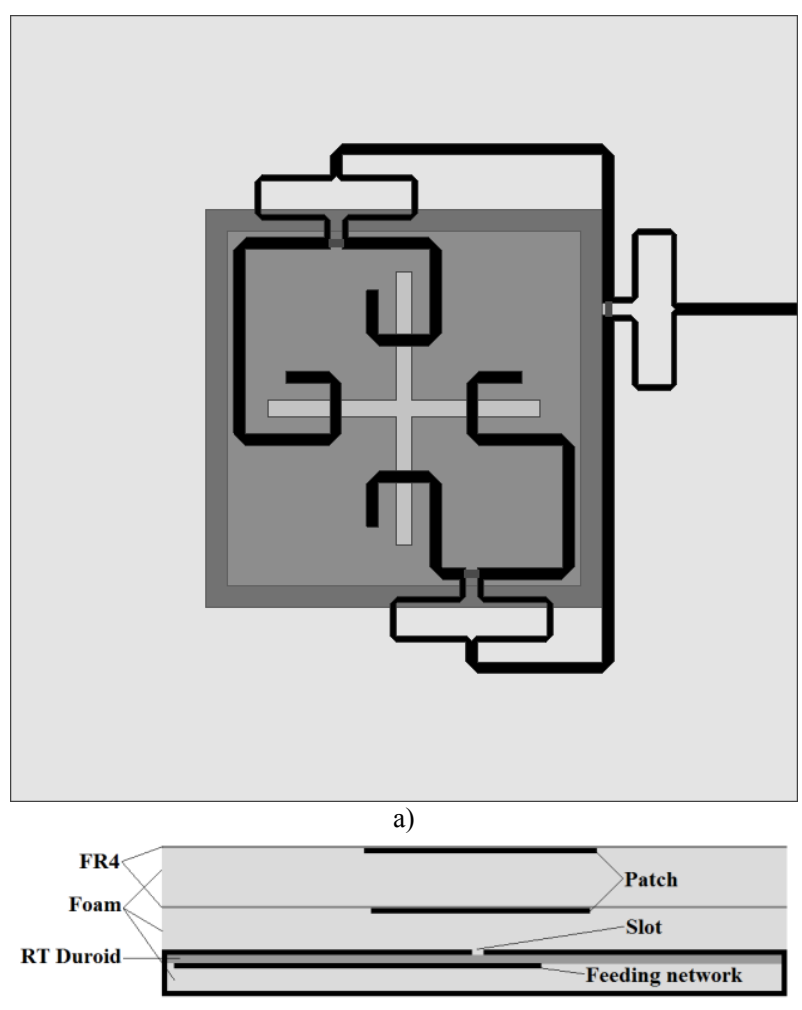

b)

Fig. 4. A cavity-backed stacked-patch antenna model. a) The top view, including the feeding network (black), the patch elements (dark grey) and the slot (light grey). b) Cross section view. 
The results are shown in Fig. 5. The measured S11 is better than $-17 \mathrm{~dB}$ along the whole required frequency band, and the measured gain and axial ratio are better than $7.4 \mathrm{dBi}$ and $1.6 \mathrm{~dB}$, respectively. The measured antenna beamwidth is between $62^{\circ}$ and $72^{\circ}$. The overall measured $10-\mathrm{dB}$ impedance bandwidth is $40 \%$ and the measured $\mathrm{AR}$ is smaller than $3 \mathrm{~dB}$ over a bandwidth of $32 \%$. The total size of the antenna is $100 \times 100 \times 12 \mathrm{~mm}^{3}$ $\left(0.67 \lambda 0 \times 0.67 \lambda 0 \times 0.08 \lambda_{0}\right.$, where $\lambda_{0}$ is a free-space wavelength at $2 \mathrm{GHz}$ ). The total weight of the antenna is $49 \mathrm{~g}$.

\section{ELECTRIC FIELD DistRIBUTION INSIDE THE CAVITY}

The metallic cavity enclosing the feeding substrates is resonant at certain frequencies and it is necessary to ensure that the resonant modes do not appear inside the operating bandwidth of the antenna. For a feeding substrate dimension of $100 \times 100 \times 2.5 \mathrm{~mm}^{3}$, which corresponds to a $1 \mathrm{U}$ CubeSat face, the first two resonant modes appear at 1.93 and $3.05 \mathrm{GHz}$, and do not compromise the antenna performance.

To better understand the effect of the pin placement and substrate shielding on the reflection coefficient, the electric field distribution inside the cavity is examined. A 4-port antenna model was used in the simulations in the same manner as previously described, and active S-parameters were calculated. Some characteristic examples of the field distribution inside the cavity, and the corresponding active Sparameters, are shown in Fig. 6 for illustration.

It is seen that a low intensity of the electric field in the centre of the cavity (close to the slot) results in a poor coupling to the patch, and vice versa. In the last case, seen in Fig. 6(c), where four shorting pins were placed in addition to the shield, the resonant frequency of the structure has increased and appears within the operating range. Here, the plotted field distribution is calculated at the resonant frequency and the field strength is an order of magnitude higher than at the other frequencies.

The cavity modes are evident in the measured $\mathrm{S}_{11}$ curves, shown in Fig. 5(c). The model with the pin wall has a lower resonant frequency $(1.84 \mathrm{GHz})$ since the electric field is fringing between the pins, increasing the effective size of the cavity. Similarly, the effective cavity size of the model with the pins and the conductive tape is smaller, resulting in a higher resonance $(1.94 \mathrm{GHz})$. These resonances are not always visible in the simulation results due to the selection of the interpolating frequency-sweep technique used in the EM software.

A good practice would be to allow for a safety margin between the cavity mode and the lowest operating frequency of the antenna. To ensure this, it is important to use the whole available surface in the interior of the satellite for the feeding structure, as this will yield a lower resonant frequency. If the antenna is to be mounted on a $3 \mathrm{U}$ CubeSat and placed on a long face, the cavity can be further enlarged. Otherwise, a higher-permittivity dielectric can be used for the feeding substrate, which would eventually produce a similar effect.
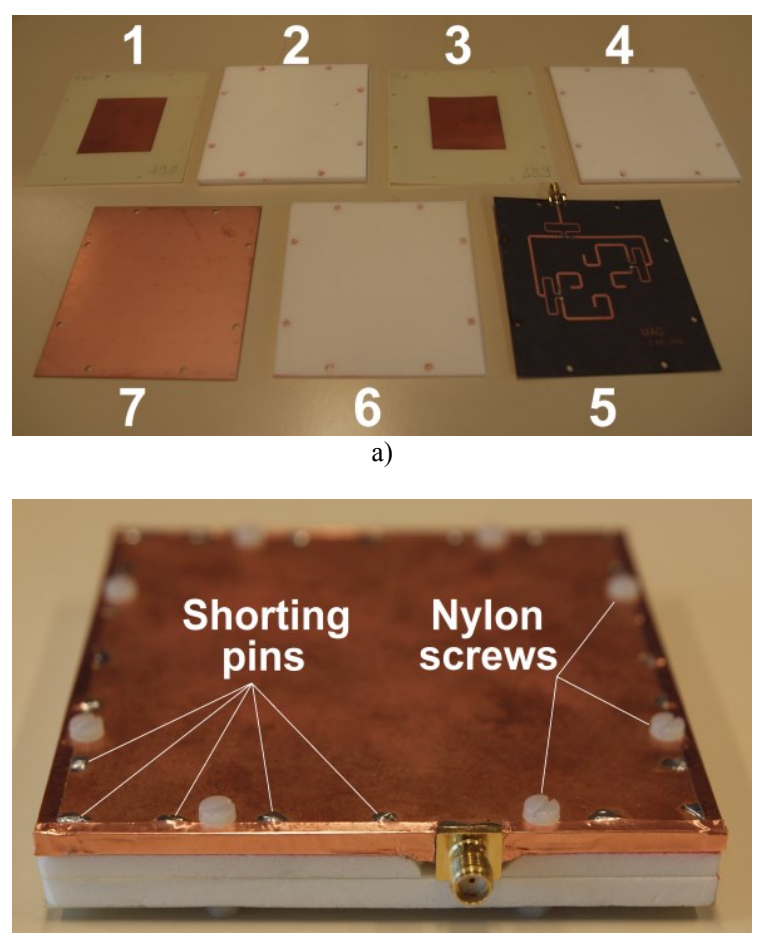

b)

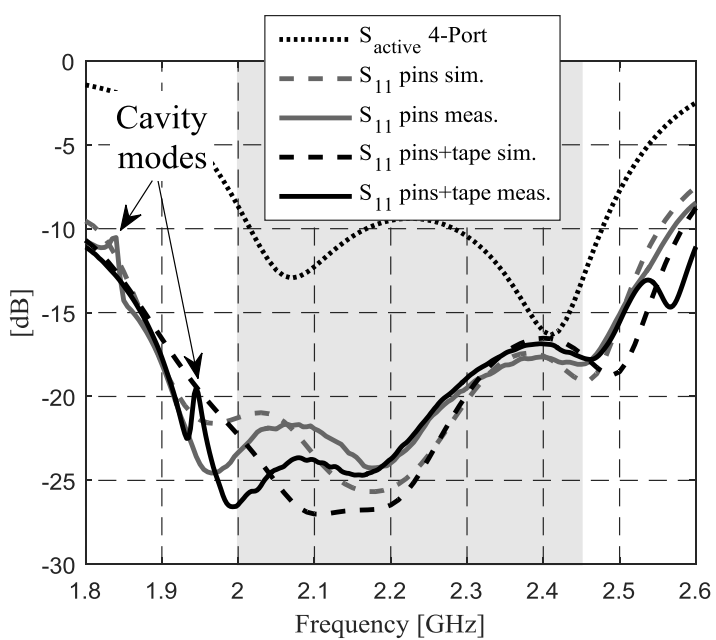

c)

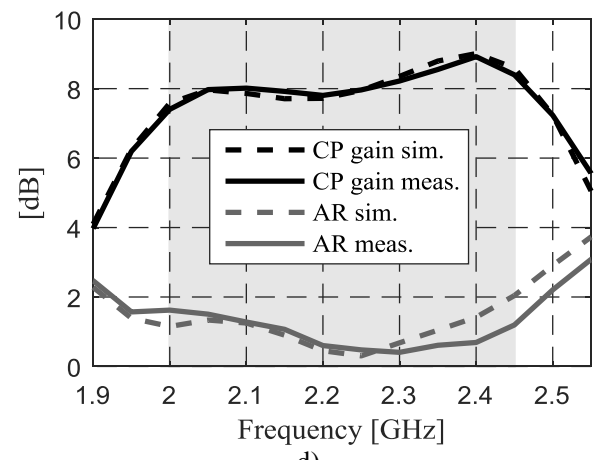

d)

Fig. 5. A cavity-backed stacked-patch antenna. a) The individual layers of the prototype. b) The realized prototype with a conductive tape around the feeding asymmetric stripline. c) The reflection coefficient. d) The antenna gain and axial ratio. 

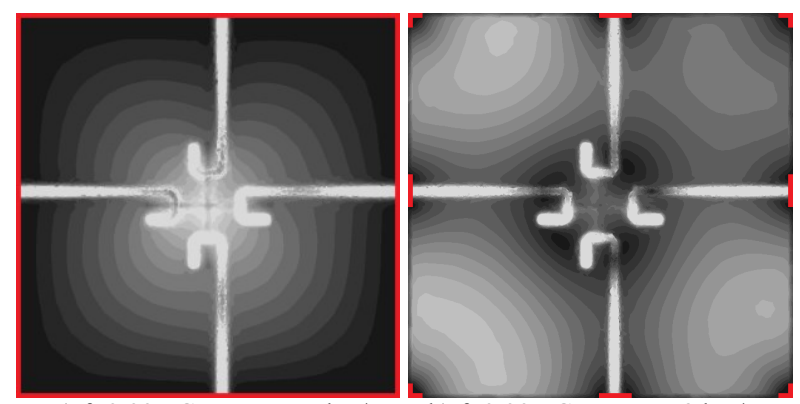

a) $\mathrm{f}=2.225 \mathrm{GHz}, \mathrm{E}_{\max }=5 \mathrm{kV} / \mathrm{m}$
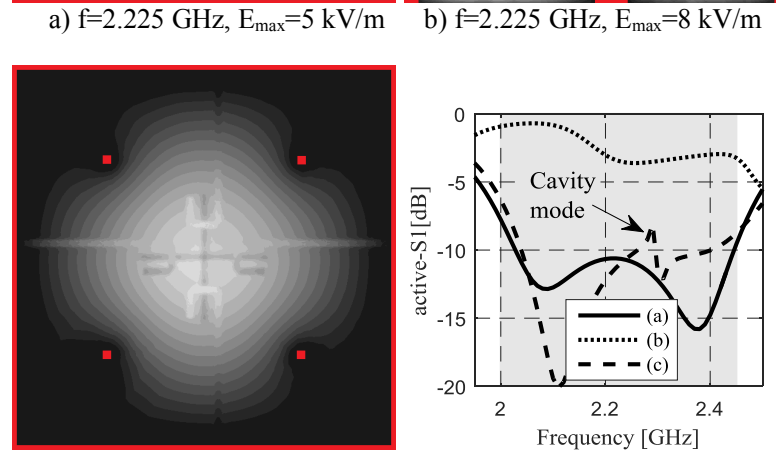

d)

Fig. 6. a) - c) Electric field distribution inside the feeding cavity of the stacked-patch antenna (4-port model) and d) the corresponding active S-parameters. The white colour represents a higher electric field strength. The red color denotes the locations where the two ground planes are electrically connected.

\section{CONCLUSIONS}

A step-by-step design process of a low-profile wideband antenna for CubeSat applications is presented. A preliminary microstrip-fed aperture-coupled patch antenna was modelled and verified through a prototype. The drawbacks of this design were addressed and improved through a set of modifications, making the antenna suitable for small satellite applications. The final design is a stacked-patch antenna, backed by an asymmetric-stripline cavity. A fabricated prototype exhibits a gain higher than $7.4 \mathrm{dBi}$ throughout the specified bandwidth, a 10-dB impedance bandwidth of $40 \%$, and a 3-dB AR-bandwidth of $32 \%$, satisfying all of the design criteria.
The next steps are the antenna placement and integration on the CubeSat, and the analysis of the antenna in a CubeSat environment.

\section{ACKNOWLEDGEMENT}

The work presented in this contribution was funded by the Swiss Commission for Innovation and Technology, in project 18328.1 CubeSatCom. In the frame of this project, we would like to acknowledge our fruitful discussion with Federico Belloni on antenna requirements.

\section{REFERENCES}

[1] CubeSat Design Specification Rev. 13, The CubeSat Program, Cal Poly SLO.

[2] Kulu, E, Nanosatellite \& CubeSat database. Internet: http://www.nanosats.eu/index.html\#figures [Oct. 2017].

[3] Constantine Kakoyiannis and Philip Constantinou (2011). Electrically Small Microstrip Antennas Targeting Miniaturized Satellites: the CubeSat Paradigm, in Microstrip Antennas, Prof. Nasimuddin Nasimuddin, InTech, DOI: 10.5772/14947, pp. Available: https://www.intechopen.com/books/microstrip-antennas/electricallysmall-microstrip-antennas-targeting-miniaturized-satellites-thecubesat-paradigm

[4] Gao, S., et al., "Antennas for small satellites", Proceedings of the 2008 Loughborough Antennas and Propagation Conference, Loughborough, UK, Mar. 2008, pp. 66-69.

[5] Nascetti, A., Pittella, E., Teofilatto, P., Pisa, S., "High-Gain S-band Patch Antenna System for Earth-Observation CubeSat Satellites", IEEE Antennas and Wireless Propagation Letters, Vol. 14, pp. 434437, 2015.

[6] Bellion, A., Elis, K., De Gaetano, S., "New compact S-band antenna for Nanosatellite TeleMetry and TeleCommand applications - EyeSat program", European Conference on Antennas and Propagation (EuCAP 2016), Apr. 10-15, Davos, 2016.

[7] Ansbro A.P., Crozzoli M., Gianola P., "Design of Circular Polarized Printed Antennas in L band", 10th International Conference on Antennas and Propagation, pp. 314-317, Apr. 1997.

[8] Zürcher, J.-F., Gardiol, F.E., Broadband Patch Antennas, Artech House, 1995, pp.55.

[9] Brachat, P., Baracco, J. M., “ Dual-Polarization Slot-Coupled Printed Antennas Fed by Stripline", IEEE Transactions on Antennas and Propagation, Vol. 43, No. 7, pp.738-742, July 1995. 